\title{
Dynamic Behavior of a Prestressed Concrete Bridge with a Switching Crack Subjected to Moving Trains
}

\author{
Chunyu Fu \\ College of Civil and Transportation Engineering, Hohai University, Nanjing, China \\ Correspondence should be addressed to Chunyu Fu; fuyupiece@163.com
}

Received 28 June 2016; Accepted 25 July 2016

Academic Editor: Yan-Wu Wang

Copyright ( 92016 Chunyu Fu. This is an open access article distributed under the Creative Commons Attribution License, which permits unrestricted use, distribution, and reproduction in any medium, provided the original work is properly cited.

\begin{abstract}
Concrete bridges chronically exposed to the natural environment are vulnerable to cracking. Under prestressing forces, the crack may close, but it will open under large dynamic loads, such as heavy trains. So the crack state can switch during the vibration. This paper investigates the nonlinear dynamic behavior of the prestressed concrete bridge with such a switching crack subjected to moving trains. Firstly, the finite element method is adopted to formulate the motion equations of train-bridge system while the crack state remains constant. Then the displacement components are analyzed to investigate the effect of static loads at the switching instant. Finally, an iterative algorithm is adopted to calculate the nonlinear responses. The proposed method is verified by the calculation of an actual prestressed concrete bridge, and the results show that the crack switching can increase the peak of the bridge displacement during the vibration, cause an obvious nonlinearity between the displacement and the vehicle mass, and make the displacement become more variable with the train velocity.
\end{abstract}

\section{Introduction}

Prestressed concrete bridges have found wide applications in railway engineering in recent years. Because of being chronically exposed to the natural environment, they are vulnerable to cracking under heavy trains, seismic excitation, and other loads. When the bridges are subjected to the independent action of static loads, especially prestressing forces, the cracks may be closed. However, if large dynamic loads, such as heavy trains, are present, the cracks will open and close in time depending on the structural vibration amplitude [1]. Various studies over the last decade have shown that a structure with such cracks exhibits nonlinear dynamic behavior [2], and its safety and serviceability are seriously affected. So it is essential to study the vibration of the prestressed concrete bridge with such cracks under moving trains.

In recent years, the vibration of cracked structures subjected to dynamic loads has attracted more and more attentions of researchers, and many methods for analyzing the vibration were proposed. Generally, these methods can be grossly divided into two kinds according to crack models used.

The first kind is the modal method for the structure with switching cracks, which are either fully open or fully closed and can switch their state instantaneously, showing a bilinear behavior. The switching condition is assumed to be determined by the sign of the normal strain [3], the displacement [4], or the curvature [5] near the crack tip. When the crack is fully open, it may seriously affect the local stiffness and response of the beam. Its effect can be modeled by means of a rotational spring model [6] or a crack disturbance function $[7,8]$. The overall behavior of the structure can be considered as a sequence of linear states, each of which can be evaluated through a modal analysis [5].

Through the modal method, Law and Zhu [9] built an interaction model between the cracked beam and a moving vehicle, which was validated by an experimental test performed on a reinforced concrete beam with a T-section. Jaksic et al. [10], respectively, calculated the eigenvalues of open and closed crack states to formulate the vibration of cracked bridges under a moving oscillator and concluded that 
the statistical properties of the bridge response were sensitive to the presence of switching crack. Fu [11] investigated the effect of switching cracks on the vibration of a continuous beam bridge subjected to moving vehicles and found that, compared to open cracks, switching cracks could result in higher acceleration and increase the high modal contribution to the displacement.

The second kind of method is mainly concerned with the structure with breathing cracks, for which there is a smooth transition phase between open and closed crack states. So the cracks can open and close continuously, and the crack state is assumed to be dependent on the responses, such as the contact condition at the crack interfaces [12], the time [13], or the curvature [1]. As the structural stiffness can vary with the crack state, it will change gradually during the vibration, and the structural behavior will exhibit obvious nonlinearity.

Ariaei et al. [1] directly used a discrete element technique to calculate the vibration of a beam with open and breathing cracks subjected to a moving mass and concluded that a beam with a breathing crack had less deflection compared to a beam with an open crack. Nguyen [14] adopted the finite element method to analyze the structural stiffness change before and after the breathing and used the instantaneous frequency of the responses under a moving vehicle to discriminate between open and breathing cracks. Andreaus et al. [2] used two-dimensional finite elements to solve the vibration of a cantilever beam with a breathing crack under the harmonic loads and impulsive loads and simulated the behavior of the crack as a frictionless contact problem.

From the aforementioned researches, it is seen that all these methods could analyze the structures with switching or breathing cracks and take the effect of dynamic loads into account. But there are few methods considering the effect of static loads. Most of researches about the vibration of prestressed concrete beams focused on the effect of prestressing force on the modal parameters. For example, Saiidi et al. [15], Kanaka and Venkateswara [16], and Miyamoto et al. [17] thought that the presence of prestressing forces decreases the natural frequencies due to the "compression softening" effect. But Hamed and Frostig [18] and Jaiswal [19] held the view that prestressing has no or negligible effect on the natural frequencies of prestressed beams.

For the prestressed concrete bridge with a switching crack, static loads including prestressing forces and the selfweight loading still persist during the vibration and may change the bridge time-history responses. It is because the sum of static and dynamic responses decides the state of the switching crack which finally affects the structural stiffness. Therefore, in this study, the dynamic behavior of the prestressed concrete bridge with a switching crack will be studied under a moving train. Firstly, motions equations of trainbridge system are firstly formulated through the finite element method; then the response components are analyzed at the switching instant to investigate the effects of static loads, and an iterative algorithm is adopted to obtain the bridge responses.

\section{Vibration of a Prestressed Concrete Bridge with a Switching Crack}

2.1. Model of Train-Bridge System. As shown in Figure 1, a prestressed concrete bridge with simple supports is considered. The axial direction of the bridge is taken as the $x$-axis and the vertical direction as the $y$-axis. It is assumed that the bridge mass and cross section are uniform along the $x$-axis, and all deformations are small enough that an orthogonal coordinate system can be used.

Suppose a switching crack appears on the bottom of the beam and its state depends on the horizontal normal strain $\varepsilon_{t c}$ at the crack region [3]:

$$
\varepsilon_{t c}=\left.\left(u_{t 0, x}-y_{c} w_{t, x x}\right)\right|_{x=l_{c}},
$$

where $u_{t 0}$ denotes the total horizontal displacement of the bridge at its centroid, $w_{t}$ is the total vertical displacement of the bridge, $y_{c}$ is the eccentricity of the bottom edge measured downwards from the section centroid, $l_{c}$ is the distance from the mid-point of the crack to the left-hand support, and ()$_{x}$ denotes differentiation with respect to $x$. The crack opens if $\varepsilon_{c}$ is positive; otherwise, it closes.

When the bridge is subjected to only static loads, including the initial prestressing force and the self-weight loading, the crack is closed as the value of the negative strain caused by the prestressing force is bigger than that of the positive strain produced by the self-weight loading, as shown in Figure 2(a).

Assume a train enters the bridge at a constant velocity $v$ from the left-hand support. When the two wheels simultaneously move on the bridge and are close to the mid-span, as shown in Figure 2(b), the crack may open as the strain produced by the train reaches its positive maximum at this moment, whose value is bigger than that of the strain sum under static loads. However, when the wheels approach the supports, as shown in Figure 1, the crack may close as the strain becomes smaller.

Therefore, while the train is moving on the bridge, the crack alternates between the closed and open states. As the structural stiffness changes with the crack state, the bridge will show different behaviors for the closed and open states.

\subsection{Motion Equations of Train-Bridge System When the Crack} Is Closed. If $\varepsilon_{c}$ is negative, the switching crack is closed, and the bridge behaves like an uncracked bridge. Here, the finite element method (FEM) is adopted to formulate the motion equations of the prestressed concrete bridge with such a crack state subjected to a moving train.

The FEM formulation will be derived via Lagrange's equations, so the potential energy and kinetic energy of the bridge should be determined firstly. During the vibration, the internal potential energy can be given as

$$
U=\int_{V_{\text {con }}} \frac{E_{\text {con }}}{2} \varepsilon_{x x}^{2} \mathrm{~d} v+\int_{V_{\text {tend }}} \frac{E_{\text {tend }}}{2} \varepsilon_{x x t}^{2} \mathrm{~d} v,
$$

where $\varepsilon_{x x}$ and $\varepsilon_{x x t}$ are the horizontal normal strains of concrete and bonded tendon, respectively; $E_{\text {con }}$ and $E_{\text {tend }}$ denote Young's modulus of concrete and tendon; and $V_{\text {con }}$ and $V_{\text {tend }}$ denote the volumes of concrete and tendon. 


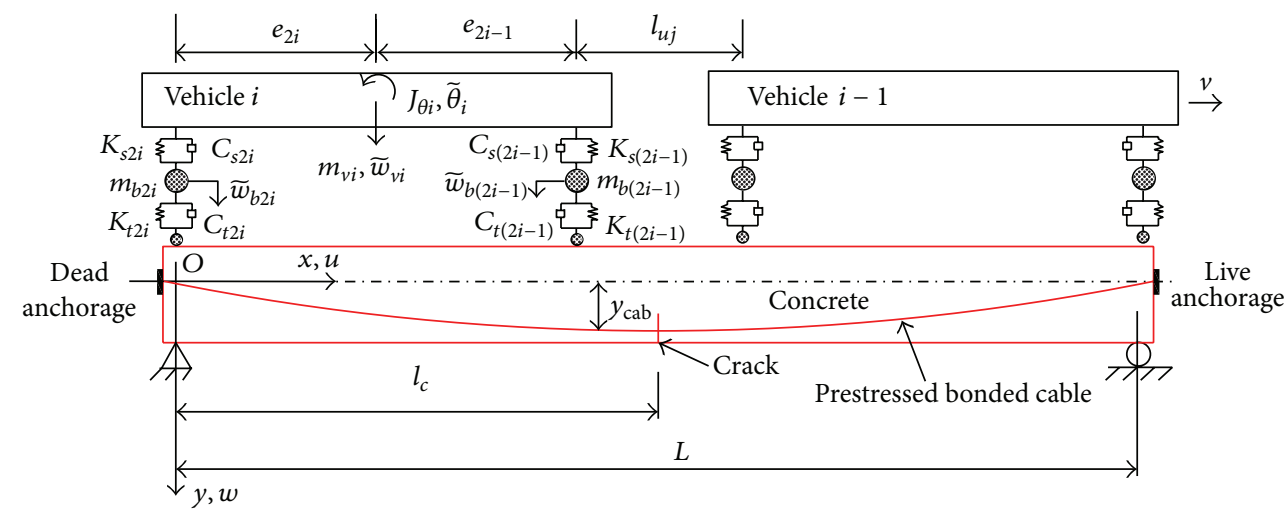

FIGURE 1: The prestressed concrete bridge subjected to a moving train.

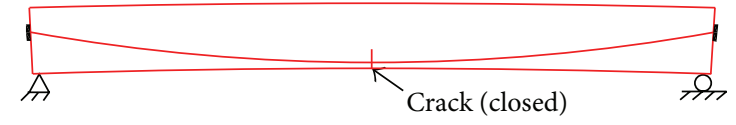

(a)

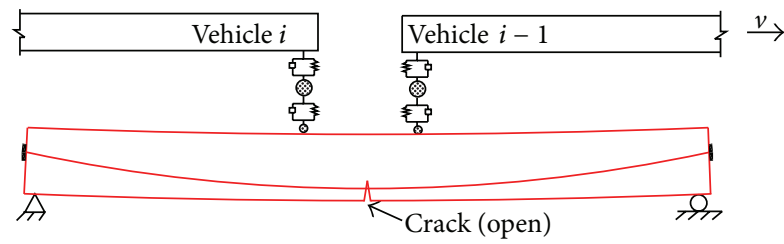

(b)

FIGURE 2: The crack state of the bridge under different loads: (a) under the prestressing force and the self-weight loading and (b) under the combined action of static loads and a moving train.

Based on the kinematic relations between the small displacements and the strains of bridge, $\varepsilon_{x x}$ and $\varepsilon_{x x t}$ can be written as

$$
\begin{gathered}
\varepsilon_{x x}(x, y, t)=u_{d 0, x}-y w_{d, x x}, \\
\varepsilon_{x x t}\left(x, y_{\text {tend }}, t\right)=u_{d 0, x}-y_{\text {tend }} w_{d, x x},
\end{gathered}
$$

where $u_{d 0}$ denotes the dynamic horizontal displacement of the bridge at its centroid, $w_{d}$ is the dynamic vertical displacement of the bridge, and $y_{\text {tend }}$ denotes the tendon eccentricity measured downwards from the section centroid.

As the bonded tendon has a much smaller mass compared to the concrete bridge, its kinetic energy can be neglected, and the kinetic energy of the bridge can be expressed by

$$
T=\int_{V_{\text {con }}} \frac{1}{2} \rho\left[\left(\dot{w}_{d}\right)^{2}+\left(\dot{u}_{d}\right)^{2}\right] \mathrm{d} v,
$$

where $\rho$ is the mass density of the concrete, $(\cdot)$ denotes a derivative with respect to time, and $u_{d}$ is the horizontal displacement of the bridge, which can be written as

$$
u_{d}(x, y, t)=u_{d 0}-y w_{d, x} .
$$

The external potential energy of the bridge is caused by the damping force, the train-bridge interaction force, and

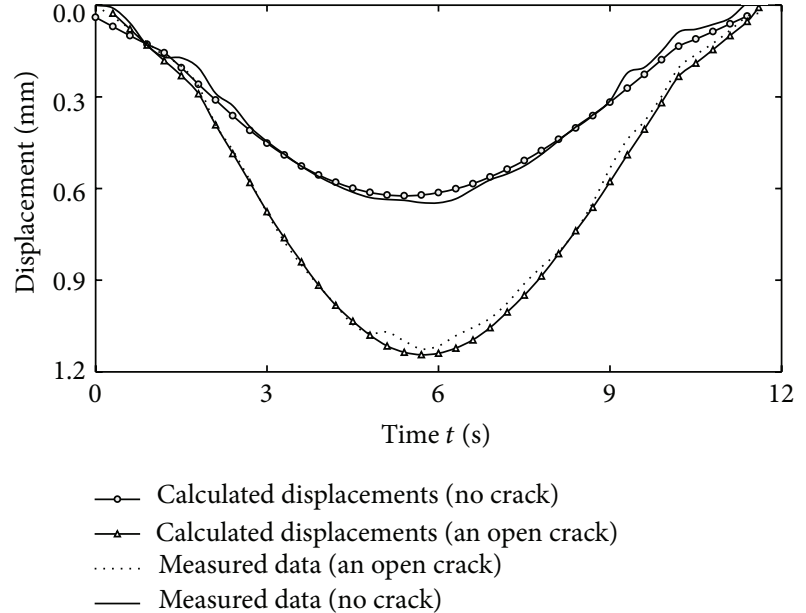

FIGURE 3: Dynamic displacements versus time at $3 / 8 L$ of a simply supported beam bridge with an open crack or no $\operatorname{crack}(v=$ $\left.0.5 \mathrm{~m} \mathrm{~s}^{-1}\right)$.

the tensile force increment of bonded tendons during the vibration, so it can be divided into three parts as follows:

$$
\begin{aligned}
V_{e 1} & =\int_{v_{\text {con }}}-c_{x} \dot{u}_{d} u_{d}-c_{y} \dot{w}_{d} w_{d} \mathrm{~d} v, \\
V_{e 2} & =\sum_{i=1}^{2 N_{v}} P_{v i} w_{d}\left(\widetilde{x}_{i}, t\right), \\
V_{e 3} & =\sum_{j=1}^{N_{\text {tend }}} P_{c x j} u_{d}\left(\hat{x}_{j}, y_{\text {tend } j}, t\right)+P_{c y j} w_{d}\left(\hat{x}_{j}, t\right),
\end{aligned}
$$

where $c_{x}$ and $c_{y}$ are the horizontal and vertical damping coefficients, $N_{v}$ is the number of the vehicles travelling on the bridge, $\tilde{x}_{i}$ is the location of $P_{v i}, \widehat{x}_{j}$ are the $x$ coordinate of the $j$ th point where the slope of cable shape changes, $y_{\text {tend } j}$ is the tendon eccentricity at the $j$ th point, and $N_{\text {tend }}$ is the number of the change points. $P_{c y j}$ and $P_{c x j}$ are the vertical and horizontal force components caused by the tension increment 
of bonded tendon at the $j$ th point during the vibration and can be expressed as

$$
\begin{aligned}
& P_{c y j}=P_{c j}\left(\sin \theta_{c j}^{-}-\sin \theta_{c j}^{+}\right), \\
& P_{c x j}=P_{c j}\left(\cos \theta_{c j}^{-}-\cos \theta_{c j}^{+}\right), \\
& \theta_{c j}^{-}=\arctan \left(\left.y_{\text {tend } j, x}\right|_{x=\widehat{x}_{j}^{-}}\right), \\
& \theta_{c j}^{+}=\arctan \left(\left.y_{\text {tend } j, x}\right|_{x=\bar{x}_{j}^{+}}\right),
\end{aligned}
$$

where $\widehat{x}_{j}^{-}$is equal to $\hat{x}_{j}-\delta x$ and $\hat{x}_{j}^{+}$is equal to $\widehat{x}_{j}+\delta x$, where $\delta x$ is a positive infinitesimal.

Assume that the bridge is divided into $n$ elements by FEM and the tendon shape in each element is assumed to be linear even if the overall shape is curvilinear. Therefore, for the curvilinear cable, the change points of the slope are the nodes of the elements. With the aid of Hermite interpolation function [20], the displacements can be represented by the displacements of its node:

$$
\begin{aligned}
& \mathbf{u}_{d}=\left[\begin{array}{c}
w_{d} \\
u_{d 0}
\end{array}\right]=\left[\begin{array}{l}
\mathbf{N}_{w}(\xi) \\
\mathbf{N}_{u}(\xi)
\end{array}\right]\left[\begin{array}{c}
\mathbf{u}_{d(i-1)} \\
\mathbf{u}_{d i}
\end{array}\right] \\
& \mathbf{N}_{w}(\xi)=\left[\begin{array}{llllll}
1-3 \xi^{2}+2 \xi^{3} & \left(\xi-2 \xi^{2}+\xi^{3}\right) l & 0 & 3 \xi^{2}-2 \xi^{3} & \left(-\xi^{2}+\xi^{3}\right) l & 0
\end{array}\right], \\
& \mathbf{N}_{u}(\xi)=\left[\begin{array}{lllllll}
0 & 0 & 1-\xi & 0 & 0 & \xi
\end{array}\right] \text {, } \\
& \mathbf{u}_{d i}(t)=\left[\begin{array}{lll}
w_{d i} & \theta_{d i} & u_{d 0 i}
\end{array}\right]^{T},
\end{aligned}
$$

where $\mathbf{N}_{w}(\xi)$ and $\mathbf{N}_{u}(\xi)$ are the Hermite interpolation functions, $l$ denotes the length of the element, $\xi$ is equal to $(x-$ $\left.x_{i-1}\right) / l, \mathbf{u}_{i}(t)$ denotes the node displacement of the beam, and $w_{d i}, \theta_{d i}$, and $u_{d 0 i}$ are the vertical displacement, sectional rotation, and horizontal displacement at the section centroid of the $i$ th node.

The general dynamic equation can be obtained from Lagrange's equations:

$$
\frac{\mathrm{d}}{\mathrm{d} t}\left(\frac{\partial T}{\partial \dot{\mathbf{u}}_{d i}}\right)-\frac{\partial T}{\partial \mathbf{u}_{d i}}+\frac{\partial\left(U-V_{e 1}-V_{e 2}-V_{e 3}\right)}{\partial \mathbf{u}_{d i}}=0 .
$$

After substituting (2)-(8d) into (9), the following motion equations of the bridge can be obtained at each instant:

$$
\begin{gathered}
\mathbf{M}_{\mathrm{con}} \frac{\mathrm{d}^{2} \mathbf{U}_{d}}{\mathrm{~d} t^{2}}+\mathbf{C}_{\mathrm{con}} \frac{\mathrm{d} \mathbf{U}_{d}}{\mathrm{~d} t}+\left(\mathbf{K}_{\mathrm{con}}+\Delta \mathbf{K}_{\mathrm{tend}}\right) \mathbf{U}_{d}=\mathbf{F}_{v}, \\
\mathbf{U}_{d}=\left[\begin{array}{lllll}
\mathbf{u}_{d 0} & \mathbf{u}_{d 1} & \cdots & \mathbf{u}_{d n}
\end{array}\right]^{T}, \\
\mathbf{F}_{v}=\sum_{i=1}^{2 N_{v}} P_{v i} \mathbf{A}_{\tilde{e}_{i}}^{T} \mathbf{N}_{w}^{T}\left(\xi_{i}\right) \\
\mathbf{A}_{\widetilde{e}_{i}}= \\
\left(3 \widetilde{e}_{i}-2\right) \text { th column } \\
{\left[\begin{array}{lllllllllllll}
0 \cdots 0 & 1 & 0 & 0 & 0 & 0 & 0 & 0 & \cdots & 0 \\
0 \cdots 0 & 0 & 1 & 0 & 0 & 0 & 0 & 0 & \cdots & 0 \\
0 \cdots 0 & 0 & 0 & 1 & 0 & 0 & 0 & 0 & \cdots & 0 \\
0 \cdots 0 & 0 & 0 & 0 & 1 & 0 & 0 & 0 & \cdots & 0 \\
0 \cdots 0 & 0 & 0 & 0 & 0 & 1 & 0 & 0 & \cdots & 0 \\
0 \cdots 0 & 0 & 0 & 0 & 0 & 0 & 1 & 0 & \cdots
\end{array}\right]_{6 \times(3 n+3)}}
\end{gathered}
$$

where $\mathbf{M}_{\text {con }}$ and $\mathbf{K}_{\text {con }}$ are, respectively, the mass and stiffness matrices contributed by the concrete and $\Delta \mathbf{K}_{\text {tend }}$ is the equivalent stiffness matrix contributed by the bonded tendons. Explicit expressions for these characteristic matrices are given in Appendix A. $\mathbf{F}_{v}$ is the nodal load of train-bridge interaction force, and $\tilde{e}_{i}$ denotes the serial number of the element in which $P_{v i}$ is located. $\mathrm{C}_{\text {con }}$ is the damping matrix, which can be represented by the Rayleigh damping matrix [21] and written as

$$
\mathbf{C}_{\text {con }}=a_{0} \mathbf{M}_{\text {con }}+a_{1} \mathbf{K}_{\text {con }},
$$

where $a_{0}$ and $a_{1}$ are the mass and stiffness proportional damping coefficients.

Assume that a train with several vehicles enters the bridge from left-hand support at a constant speed $v$ and each of the vehicles is represented by a four-degree-of-freedom model shown in Figure 1. The motion equations of the vehicle model can be derived using Lagrange formulation as follows:

$$
\mathbf{M}_{v i} \ddot{\mathbf{U}}_{v i}+\mathbf{C}_{v i} \dot{\mathbf{U}}_{v i}+\mathbf{K}_{v i} \mathbf{U}_{v i}=-\mathbf{P}_{t v i}+\mathbf{P}_{0 i},
$$

where $\mathbf{M}_{v i}, \mathbf{C}_{v i}, \mathbf{K}_{v i}$ are the mass, damping, and stiffness matrices of the vehicle, respectively, and they are given in Appendix B; $\mathbf{U}_{v i}=\left[\begin{array}{llll}\widetilde{w}_{v i} & \widetilde{\theta}_{i} & \widetilde{w}_{b 2 i} & \widetilde{w}_{b(2 i-1)}\end{array}\right]$ is the response vector of the $i$ th vehicle; $\mathbf{P}_{0 i}=\left\{m_{v i} g, 0, m_{b(2 i-1)} g, m_{b 2 i} g\right\}^{T}$ is the load vector of the vehicle weight; $\mathbf{P}_{t v i}=$ $\left\{0,0, P_{v(2 i-1)}, P_{v 2 i}\right\}^{T}$ is the vector of train-bridge interaction force, which can be expressed by

$$
\begin{aligned}
P_{v j}(t)= & K_{t j}\left[\widetilde{w}_{b j}-w\left(\widetilde{x}_{j}(t), t\right)\right] \\
& +C_{t j}\left[\dot{\tilde{w}}_{b j}-\dot{w}\left(\widetilde{x}_{j}(t), t\right)\right] \quad j=2 i-1,2 i,
\end{aligned}
$$

where $K_{t j}$ and $C_{t j}$ are the stiffness and the damping of the wheels. 
Substituting (12) and (13) into (10a)-(10d) the combined equations of the train-bridge system are obtained:

$$
\begin{aligned}
\mathbf{M}_{s} \frac{\mathrm{d}^{2} \mathbf{U}_{s}}{\mathrm{~d} t^{2}}+\mathbf{C}_{s} \frac{\mathrm{d} \mathbf{U}_{s}}{\mathrm{~d} t}+\mathbf{K}_{s} \mathbf{U}_{s} & =\mathbf{P}_{s}, \\
\mathbf{U}_{s} & =\left[\begin{array}{lllll}
\mathbf{U}_{d} & \mathbf{U}_{v 1} & \cdots & \mathbf{U}_{v N_{v}}
\end{array}\right]^{T}, \\
\mathbf{P}_{s} & =\left[\begin{array}{lllll}
0 & \mathbf{P}_{01} & \cdots & \mathbf{P}_{0 N_{v}}
\end{array}\right]^{T},
\end{aligned}
$$

where $\mathbf{M}_{s}, \mathbf{C}_{s}, \mathbf{K}_{s}$ are the characteristic matrices of the trainbridge system. Explicit expressions for these matrices are given in Appendix C.

\subsection{Motion Equations of Train-Bridge System When the Crack} Is Open. If $\varepsilon_{c}$ is positive, the crack is open and its effect can be modeled by means of the damage function designed for concrete structures, which was proposed by Abdel Wahab et al. [22]. The function completely describes the stiffness reduction around the crack region through a reduction in Young's modulus of concrete, which is expressed as follows:

$$
\begin{aligned}
E_{c}(x)=E_{\text {con }}\left\{1-\alpha \cos ^{2}\right. & {\left.\left[\frac{\pi}{2}\left(\frac{2\left|x-l_{c}\right|}{\beta L}\right)^{\eta}\right]\right\} } \\
& \text { for } l_{c}-\frac{\beta L}{2}<x<l_{c}+\frac{\beta L}{2},
\end{aligned}
$$

where $E_{c}$ denotes the modulus around the crack region when the crack is open. $\alpha, \beta$, and $\eta$ are the damage parameters of the crack and are determined by the vibration characteristics extracted from the experimental data. The first parameter $\alpha$ characterizes the magnitude of the damage, with values between 0.0 and 1.0. Parameter $\beta$ characterizes the length of the cracked region and also has values between 0.0 and 1.0. Parameter $\eta$ denotes the variation of the modulus from the center of the crack to the end of the damaged region.

So the change value of the modulus before and after the opening can be written as

$$
\begin{aligned}
\Delta E_{c}(x)=E_{\mathrm{con}} \alpha \cos ^{2}[ & \left.\frac{\pi}{2}\left(\frac{2\left|x-l_{c}\right|}{\beta L_{n}}\right)^{\eta}\right] \\
& \text { for } l_{c}-\frac{\beta L}{2}<x<l_{c}+\frac{\beta L}{2} .
\end{aligned}
$$

This change will cause a variation in the stiffness matrix of the bridge, which is written as

$$
\begin{aligned}
& \Delta \mathbf{K}_{\text {crack }}=\sum_{i=1}^{N_{\text {crack }}} \mathbf{A}_{\bar{e}_{i}}^{T} \Delta \mathbf{K}_{c \bar{e}_{i}} \mathbf{A}_{\bar{e}_{i}}, \\
& \Delta \mathbf{K}_{c \bar{e}_{i}} \\
& =\int_{0}^{1} \frac{\Delta E_{c}\left(\xi l+x_{\bar{e}_{i}-1}\right) I_{\text {con }}}{l^{3}}\left(\frac{\mathrm{d}^{2} \mathbf{N}_{w}(\xi)}{\mathrm{d} \xi^{2}}\right)^{T} \frac{\mathrm{d}^{2} \mathbf{N}_{w}(\xi)}{\mathrm{d} \xi^{2}} \\
& \quad+\frac{\Delta E_{c}\left(\xi l+x_{\bar{e}_{i}-1}\right) A_{\text {con }}}{l}\left(\frac{\mathrm{d} \mathbf{N}_{u}(\xi)}{\mathrm{d} \xi}\right)^{T} \frac{\mathrm{d} \mathbf{N}_{u}(\xi)}{\mathrm{d} \xi} \mathrm{d} \xi,
\end{aligned}
$$

where $\mathbf{u}_{\text {st } i}=\left[\begin{array}{lll}w_{\text {st } i} & \theta_{\text {st } i} & u_{\text {st } 0 i}\end{array}\right]^{T}(i=0 \sim n)$ is the static displacement vector of the bridge.

Meanwhile, the static displacement is related to not only the static loads, but also the structural stiffness. Therefore, at $t_{0}^{-}$, the crack is closed, and $\mathbf{U}_{\text {st }}$ can be expressed as

$$
\mathbf{U}_{\text {st }}\left(t_{0}^{-}\right)=\left(\mathbf{K}_{\text {con }}+\Delta \mathbf{K}_{\text {tend }}\right)^{-1} \mathbf{F}_{\text {st }} \text {, }
$$

where $\mathbf{F}_{\text {st }}$ is the static load vector caused by the initial prestressing force and the self-weight loading of the bridge.

But at $t_{0}^{+}$, the crack is open, and the stiffness changes. So $\mathbf{U}_{\text {st }}$ can be rewritten as

$$
\mathbf{U}_{\text {st }}\left(t_{0}^{+}\right)=\left(\mathbf{K}_{\text {con }}+\Delta \mathbf{K}_{\text {tend }}-\Delta \mathbf{K}_{\text {crack }}\right)^{-1} \mathbf{F}_{\text {st }} .
$$

Comparing (21) and (22), we can see that $\mathbf{U}_{\text {st }}\left(t_{0}^{+}\right)$is not equal to $\mathbf{U}_{\mathrm{st}}\left(t_{0}^{-}\right)$as the stiffness in the two equations is different. 
By substituting (20a) into (19a), we can obtain the following equation:

$$
\mathbf{U}_{d}\left(t_{0}^{+}\right)=\mathbf{U}_{d}\left(t_{0}^{-}\right)+\mathbf{U}_{\mathrm{st}}\left(t_{0}^{-}\right)-\mathbf{U}_{\mathrm{st}}\left(t_{0}^{+}\right)
$$

From (23), it is found that, compared to $\mathbf{U}_{\mathrm{st}}\left(t_{0}^{-}\right), \mathbf{U}_{\mathrm{st}}\left(t_{0}^{+}\right)$ has an added dynamic displacement, whose value is equal to the difference between the static displacements before and after the crack switching. Then $\mathbf{U}_{\mathrm{st}}\left(t_{0}^{+}\right)$can be taken as the initial displacement at $t_{0}^{+}$, and (18a) and (18b) are solved to calculate the dynamic displacement beyond $t_{0}^{+}$until the crack closes again. Finally, according to the current static displacement, the total displacement of bridge is obtained by using (20a) and (20b).

When the crack switches from the open state to the closed one, the bridge stiffness also changes and in a similar way the bridge displacements can be calculated.

As the total response of the bridge with a switching crack determines the crack state, which in turn affects the response through the structural stiffness change, the following iterative algorithm should be adopted to calculate the total displacements at the instant $t+\Delta t$ if the crack is switching:

(1) Estimate the normal strain at the crack region $\varepsilon_{t c i}(i=$ 0 ) by using the total displacements at the previous instant $t$ according to (1).

(2) Decide the crack state through $\varepsilon_{t c i}$ at $t+\Delta t$, and judge whether there is a crack switching through comparing the crack states at $t+\Delta t$ and at $t$.

(3) If there is no switching, adopt (21) and (14a)-(14c) to calculate the static and dynamic displacements, respectively, when the crack is closed; when it is open, use (22) and (18a)-(18b). If there is switching, take the added dynamic displacement into account through (23), and then use the corresponding equations to calculate the static and dynamic displacements.

(4) Calculate the total displacements at $t+\Delta t$ using (20a) and (20b), and estimate the strain $\varepsilon_{t c(i+1)}$.

(5) Compare the two latest strains estimated, and calculate whether the strains satisfy the equation as follows:

$$
\left\|\varepsilon_{t c(i+1)}-\varepsilon_{t c i}\right\|<\varepsilon
$$

where $\varepsilon$ is the permissible error.

(6) If (24) is valid, the total displacements at $t+\Delta t$ are equal to the displacements that have been just calculated; otherwise, $i$ is assigned to $i+1$, and return to step (2) to calculate again.

Because the train is moving on the bridge and its location is changing, the characteristic matrices of the train-bridge system vary with time, and (14a)-(14c) and (18a)-(18b) are time-varying differential equations, which should be solved using step-by-step integration methods. In this paper, the Houbolt method [21] is used to obtain the bridge responses.

In this solving process, the difficulties lie in the calculation of the responses at the switching instants. In order to

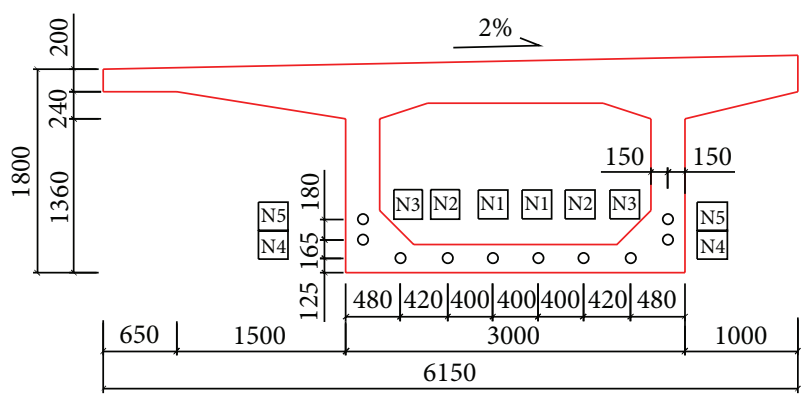

FIGURE 4: The cross section at the mid-span of the bridge (unit: $\mathrm{mm}$ ).

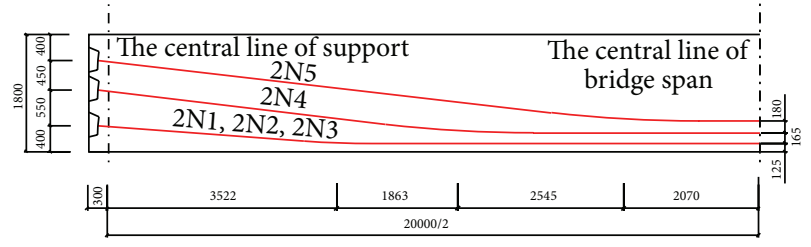

FIgURE 5: The half of bonded tendon layout (unit: $\mathrm{mm}$ ).

accurately obtain these responses, the key points for attention are given as follows:

(1) The influence of crack switching on the stiffness matrix of the train-bridge system should be taken into account.

(2) The added dynamic displacement caused by the switching needs to be calculated.

(3) The crack state estimated by the strain $\varepsilon_{t c(i+1)}$ should be identical with that estimated by $\varepsilon_{t c i}$.

\section{Examples}

4.1. Comparison with Experimental Data. To validate the proposed method for the vibration analysis of train-bridge system, the displacements of an experimental concrete beam subjected to a moving vehicle are calculated by the method, and the results are compared with the experimental data from Law and Zhu [9]. The bridge and vehicle parameters are the same with those adopted by Law and Zhu [9], and the crack is assumed to be in the open state if it is present. The compared results are shown in Figure 3.

From Figure 3, we can see that the measured and calculated displacements are close to each other, no matter whether there is an open crack or not. This agreement indicates the effectiveness of the proposed method for analyzing the vibrations of intact bridges and cracked bridges.

4.2. Dynamic Behavior of a Prestressed Concrete Bridge with a Switching Crack under a Moving Train. The bridge considered in this paper is a standard simply supported bridge used in Qian-Shen Passenger Dedicated Line of China, which is also a representative of the prestressed concrete bridges adopted for the China high-speed railway. 


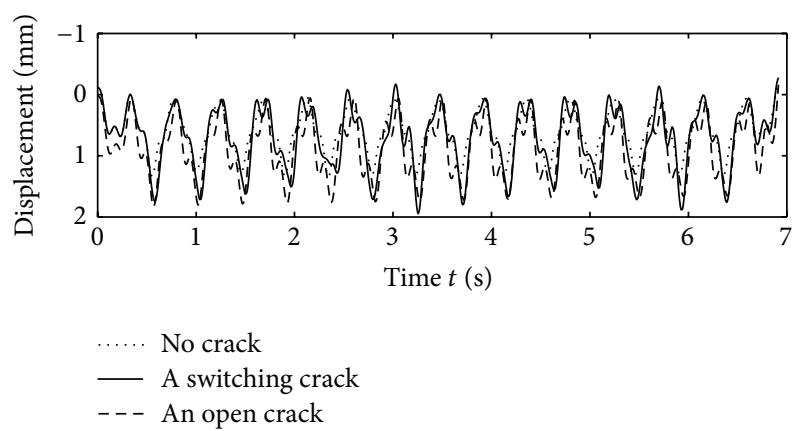

FIGURE 6: The total displacements at the mid-span of the bridges subjected to the train with a velocity of $200 \mathrm{~km} / \mathrm{h}$ when the crack type is different.

The span of the bridge is $20 \mathrm{~m}$. The cross section at the mid-span and the cable layout are shown in Figures 4 and 5, respectively. The concrete elasticity modulus is $34.5 \mathrm{GPa}$, and the secondary dead load is $80 \mathrm{kN} / \mathrm{m}$. Every bonded cable is constituted of 6 steel strands, and the nominal diameter, area, elasticity modulus, and effective tension prestress of each strand are $15.2 \mathrm{~mm}, 140 \mathrm{~mm}^{2}, 195 \mathrm{GPa}$, and $970 \mathrm{MPa}$.

Germany train ICE3 is taken as a model of the train, and the vehicle number of the train is 15 . Each of these vehicles adopts the following parameters: $m_{v i}=48 \mathrm{t}$, the rotational inertia of the vehicle $J_{\theta i}=2.7 \times 10^{6} \mathrm{~kg} \mathrm{~m}^{2}, e_{2 i-1}=e_{2 i}=$ $8.688 \mathrm{~m}, K_{s(2 i-1)}=K_{s 2 i}=400 \mathrm{kN} / \mathrm{m}, C_{s(2 i-1)}=C_{s 2 i}=$ $6 \mathrm{kN} \mathrm{s} / \mathrm{m}, m_{b(2 i-1)}=m_{b 2 i}=3200 \mathrm{~kg}, K_{t(2 i-1)}=K_{t 2 i}=$ $2080 \mathrm{kN} / \mathrm{m}, C_{t(2 i-1)}=C_{t 2 i}=10 \mathrm{kN} \mathrm{s} / \mathrm{m}(i=1 \sim 20)$, and the distance between the nearest wheels of two adjacent vehicles $l_{u j}=7.4 \mathrm{~m}(j=1 \sim 19)$.

Suppose a switching crack appears at the mid-span of the bridge and the following crack parameters are used: $l_{c}=10 \mathrm{~m}, \alpha=0.487, \beta=0.1$, and $\eta=20$. Figure 6 shows the displacements at the mid-span of the bridge subjected to the train with a constant velocity of $200 \mathrm{~km} / \mathrm{h}$. To illuminate the different dynamic behavior of this studied bridge, its displacements are compared with those of two other bridges, which have the same bridge properties with the studied bridge, except in terms of crack type, as shown in Figure 6.

From Figure 6, it is seen that all three bridges almost vibrate periodically with the train moving. Their displacements reach the positive peaks if the two wheels simultaneously move on the bridge and are close to the mid-span, while they reach the negative ones if only one wheel moves on the bridge and approaches the supports. However, the maximum value of the peaks is largest when the crack is switching. The reason for this may be found from the change of crack state during the vibration.

Compared to the constant stiffness of the bridge with an open crack or no crack, the stiffness of the bridge with a switching crack changes with the crack state, as shown in Figure 7(a). As a result, at every switching instant, the static displacements caused by the static loads shift, an added dynamic displacement appears, and the dynamic displacement changes suddenly, as shown in Figure 7(b).

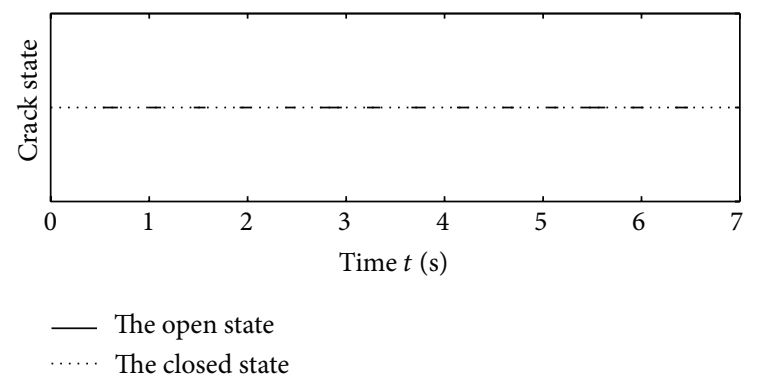

(a)
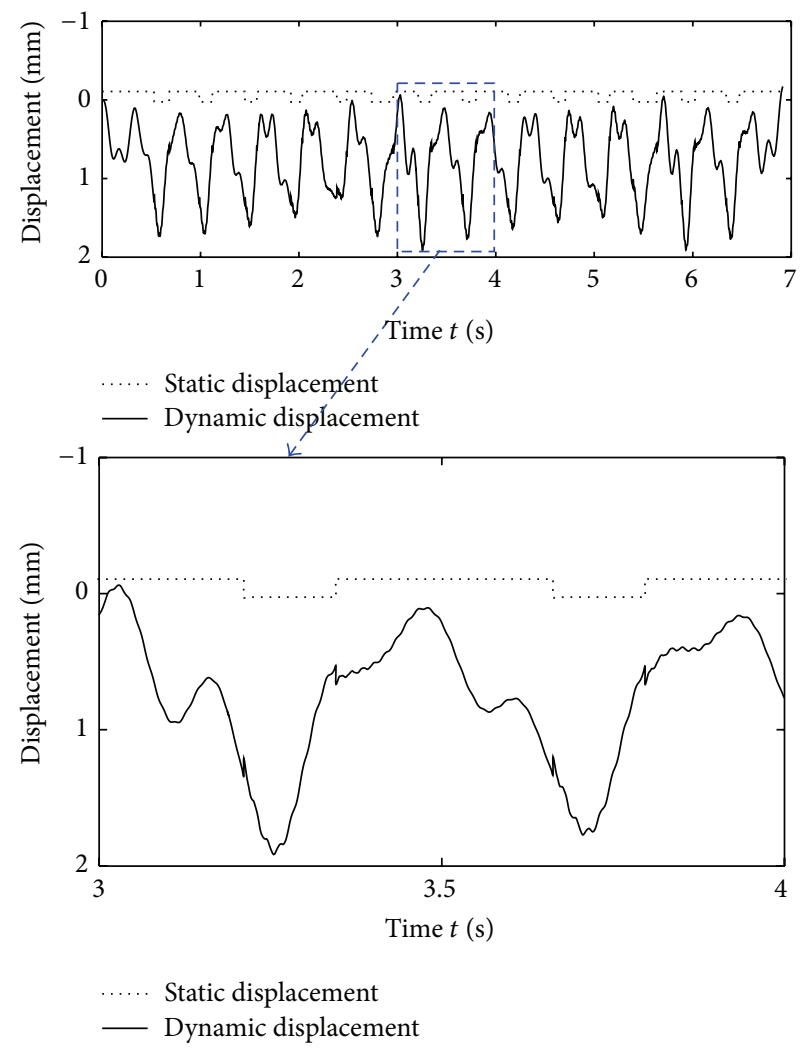

(b)

FIGURE 7: The responses of the bridge with a switching crack subjected to the train with a velocity $200 \mathrm{~km} / \mathrm{h}$ : (a) the crack states and (b) the displacement components.

The added displacement can be viewed as an initial displacement at the switching instant, which will cause a free vibration of bridge at itself natural frequency. So beyond the instant, the added displacement can cause positive displacements and negative displacements during vibration. If the total displacement nearly reaches its peak and the displacement caused by the added displacement has the same sign with the dynamic displacement, the peak value will be increased. For example, at the opening instant $3.21 \mathrm{~s}$ shown in Figure 7(b), the dynamic displacement decreases due to the appearance of the negative added displacement. However, at $3.26 \mathrm{~s}$, the dynamic displacement reaches its positive peak, and the added displacement causes a positive displacement. It is because of the fact that there is about a third of the 


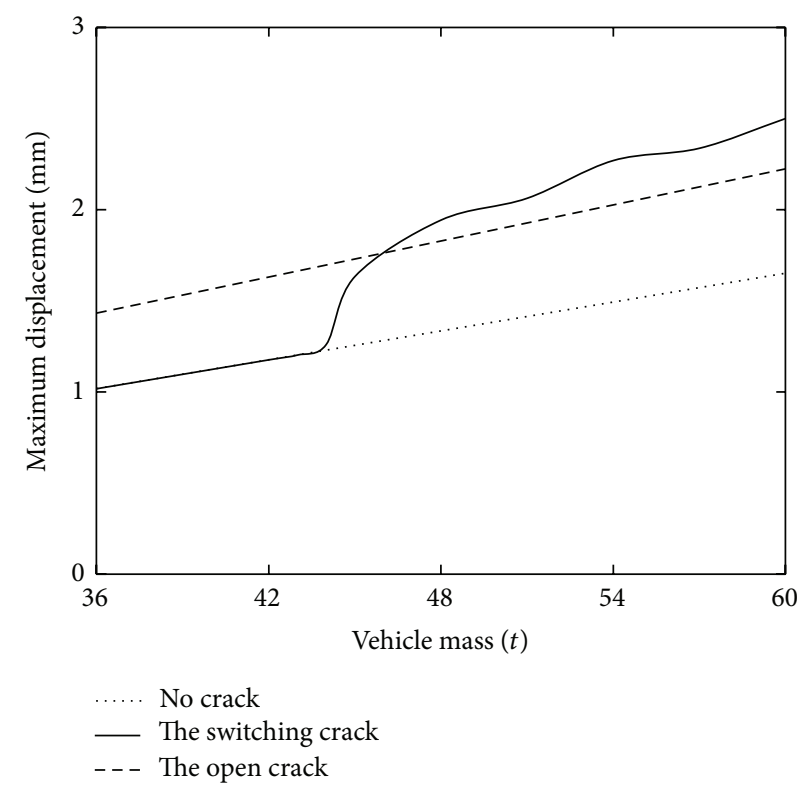

FIGURE 8: The effect of vehicle mass on the maximum of the total displacement at the mid-span of the bridge.

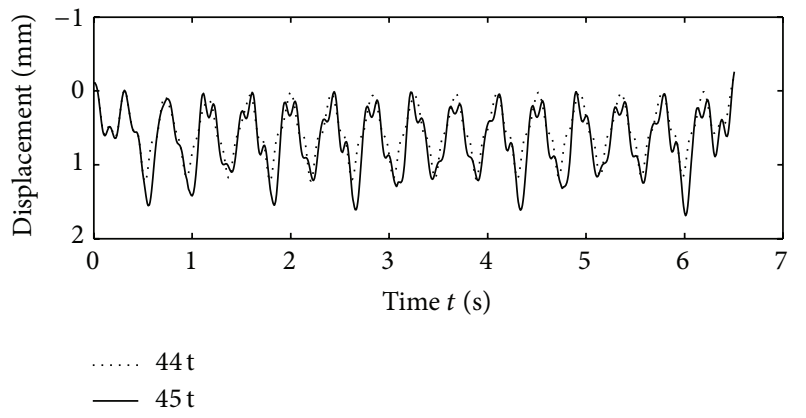

(a)

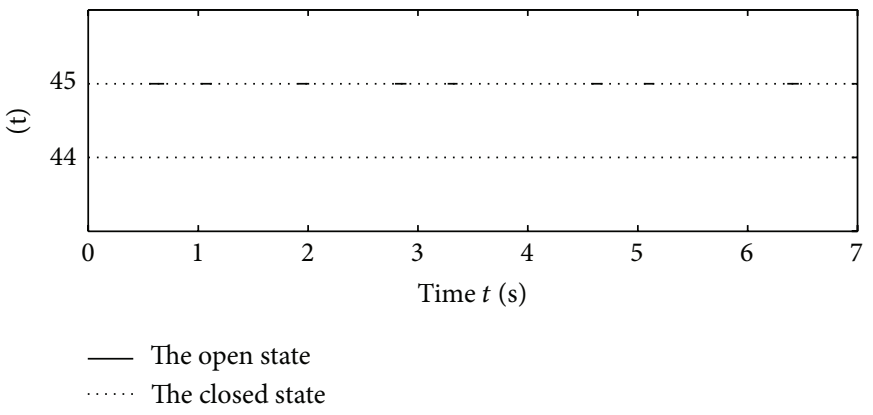

(b)

FIGURE 9: The responses of the bridge with a switching crack when the vehicle mass is different: (a) the total displacement at the mid-span of the bridge and (b) the crack states.

bridge fundamental period between $3.21 \mathrm{~s}$ and $3.26 \mathrm{~s}$, during which the free vibration caused by the added displacement can change its displacement from a negative minimum to a positive value. Finally, the peak value of total displacement is increased, and the bridge vibration is intensified by the crack switching.

4.3. Effect of the Vehicle Mass on the Dynamic Behavior. The actual train moving on the bridge possesses a random vehicle mass. Here assume the trains with different masses travel through the bridge mentioned in Section 4.2. The vehicle mass is changed from $36 \mathrm{t}$ to $60 \mathrm{t}$, but the train velocity is held at $200 \mathrm{~km} / \mathrm{h}$. The effect of the mass on the maximum displacement is investigated in Figure 8. It is observed that when the vehicle is light, the bridge with a switching crack has the same displacement with the bridge with no crack. It is because the light vehicles, such as the vehicle with a mass of $44 \mathrm{t}$ shown in Figure 9, produce a small normal tensile strain, which could not neutralize the compressive strain caused by static loads, including the initial prestressing force and the self-weight loading. So the normal strain at the crack region is still negative, and the switching crack remains closed during the vibration, which makes the bridge with a switching crack behave like an intact bridge.

However, when the vehicle becomes heavier, such as the vehicle having a mass of $48 \mathrm{t}$ shown in Figure 6 , the moving train will cause a bigger tensile strain, which makes the normal strain at the crack region alternate between the positive value and the negative one. So the crack will switch its state during the vibration and increase the bridge displacement. As a result, the heavier vehicle produces a larger maximum displacement when the crack is switching than when it is open.

From Figure 8, it is seen that in the relationship between the maximum displacement and the vehicle mass there is a sudden change when the mass is equal to $44 \mathrm{t}$. It is due to the fact that the crack remains closed if the mass is less than or equal to $44 \mathrm{t}$, while it can switch if the mass is bigger than $44 \mathrm{t}$. For example, when the mass is equal to $45 \mathrm{t}$, it is 


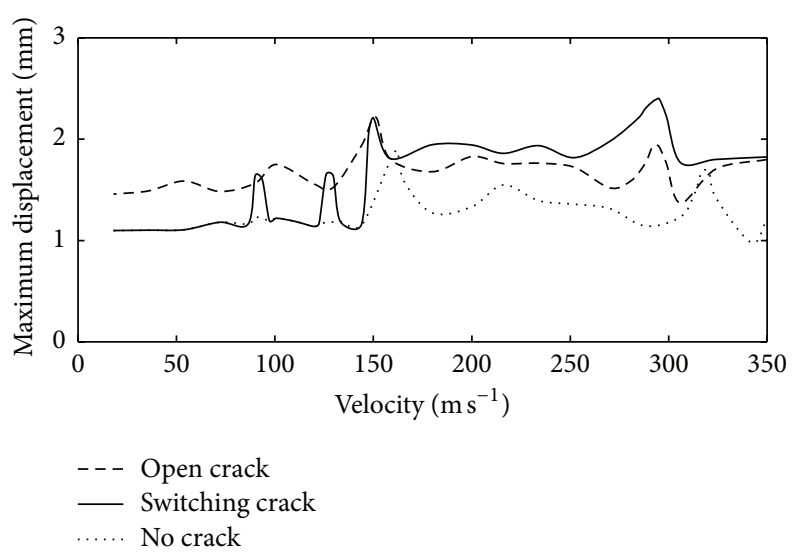

FIGURE 10: The maximum of the total displacement at the mid-span of the bridge versus the train velocity.

close to $44 \mathrm{t}$, but the vehicle can make the crack switch, as shown in Figure 9(b). So the maximum displacement changes suddenly when the mass changes from being $44 \mathrm{t}$ to $45 \mathrm{t}$, and the relationship between the displacement and the vehicle mass exhibits an obvious nonlinearity.

\subsection{Effect of the Vehicle Velocity on the Dynamic Behavior.} Assuming the vehicle mass is held at $48 \mathrm{t}$, Figure 10 analyzes the effect of velocity on the maximum displacement. It is observed that when the velocity is less than $72 \mathrm{~km} / \mathrm{h}$, the displacement of the bridge with a switching crack is the same with that of the intact bridge. When the velocity is more than $150 \mathrm{~km} / \mathrm{h}$, the displacement is biggest when the crack is switching. It is because of the fact that if the bridge is subjected to the train with a low velocity, the dynamic responses are small, and the switching crack remains closed during vibration. But if the velocity is high, the dynamic responses become bigger, and the dynamic tensile strain is large enough to neutralize the compressive strain caused by static loads. So the crack will switch with the normal strain at the crack region, and the displacement becomes larger.

When the velocity is between $72 \mathrm{~km} / \mathrm{h}$ and $150 \mathrm{~km} / \mathrm{h}$, the bridge with a switching crack behaves like an intact bridge under the train with some velocity, such as $120 \mathrm{~km} / \mathrm{h}$. But at some given velocity, such as $90 \mathrm{~km} / \mathrm{h}$, the train can cause the crack to be open for a few seconds. So the crack switches several times, and in the intervals between the switching instants the displacement is increased, as shown in Figure 11.

Therefore, once the crack can switch, the displacement is increased, and a new local maximum appears in the relationship between the displacement and the velocity, as shown in Figure 10. Compared to the bridge with an open crack or no crack, the bridge with a switching crack has a displacement that is more variable with the train velocity.

\section{Conclusions}

In this study, the nonlinear dynamic behavior of the prestressed concrete bridge with a switching crack is analyzed

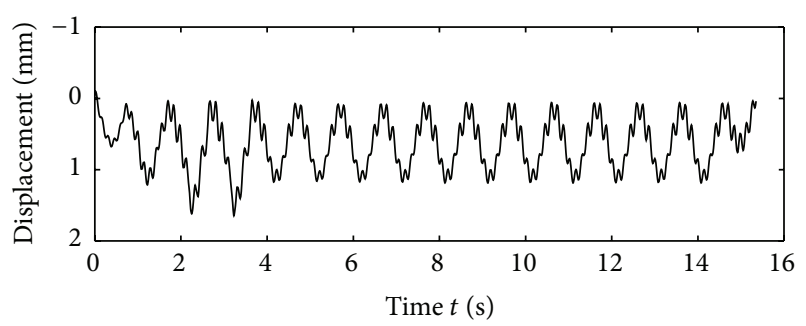

(a)

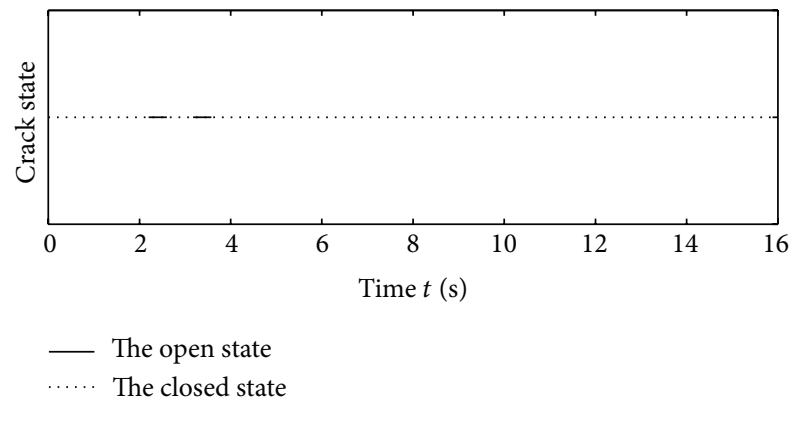

(b)

FIGURE 11: The responses of the bridge with a switching crack subject to the train with a velocity of $90 \mathrm{~km} / \mathrm{h}$ : (a) the total displacement at the mid-span of the bridge and (b) the crack state.

under moving trains. Firstly, the finite element method is adopted to formulate the motion equations of train-bridge system while the crack state remains constant. Then the displacement components are analyzed to investigate the effect of static loads at the switching instant. Finally, an iterative algorithm is adopted to calculate the bridge responses. The proposed method is verified by the calculation of an actual prestressed concrete bridge subjected to moving trains, and the following nonlinear behaviors of the bridge can be found:

(1) The crack switching can increase the bridge displacement during the vibration. It is because of the fact that the switching can cause the change of the bridge stiffness, which alters the static displacement and leads to an added dynamic displacement. This added displacement can be viewed as the initial displacement at the switching instant and will increase the peak of the displacement.

(2) The switching causes an obvious nonlinearity between the bridge displacement and the vehicle mass. This is due to the fact that the light vehicle produces a small dynamic tensile strain and makes the crack remain closed during the vibration. But the heavy vehicle produces a large strain and causes the crack to switch with the strain at the crack region. The state of switching crack changes with the vehicle mass, so does the bridge stiffness.

(3) The switching makes the displacement become more variable with the train velocity. The reason for this behavior is that under the train with low velocities the 
crack remains closed; under the train with high velocities, the crack always switches during the vibration; if the train moves at some given moderate velocity, the crack just switches several times. As the crack state is highly changeable to the train velocity, the bridge displacement becomes variable with it.

\section{Appendix}

\section{A. Explicit Expressions for the Characteristic Matrices in (10a) $-(10 d)$}

$$
\begin{aligned}
& \mathbf{M}_{\text {con }}=\sum_{e=1}^{n} \mathbf{A}_{e}^{T} \mathbf{M}_{\text {cone }} \mathbf{A}_{e}, \\
& \mathbf{K}_{\text {con }}=\sum_{e=1}^{n} \mathbf{A}_{e}^{T} \mathbf{K}_{\text {cone }} \mathbf{A}_{e}, \\
& \Delta \mathbf{K}_{\text {tend }}=\sum_{e=1}^{n} \mathbf{A}_{e}^{T} \Delta \mathbf{K}_{\text {tende }} \mathbf{A}_{e}, \\
& \mathbf{K}_{\text {cone }} \\
& \quad=\int_{0}^{1} \frac{E_{\text {con }} I_{\text {con }}+E_{\text {tend }} A_{\text {tend }} y_{\text {tend }}^{2} \sqrt{1+y_{\text {tend }, x}^{2}}}{l^{3}}\left(\frac{\mathrm{d}^{2} \mathbf{N}_{w}(\xi)}{\mathrm{d} \xi^{2}}\right)^{T} \\
& \quad \cdot\left(\frac{\mathrm{d}^{2} \mathbf{N}_{w}(\xi)}{\mathrm{d} \xi^{2}}\right)
\end{aligned}
$$

$$
\begin{gathered}
+\frac{E_{\text {con }} A_{\text {con }}+E_{\text {tend }} A_{\text {tend }} \sqrt{1+y_{\text {tend }, x}^{2}}}{l}\left(\frac{\mathrm{d} \mathbf{N}_{u}(\xi)}{\mathrm{d} \xi}\right)^{T} \\
\cdot\left(\frac{\mathrm{d} \mathbf{N}_{u}(\xi)}{\mathrm{d} \xi}\right) \mathrm{d} \xi \\
\mathbf{M}_{\text {cone }}=\int_{0}^{1} \rho A_{\text {con }} l \mathbf{N}_{w}^{T}(\xi) \mathbf{N}_{w}(\xi)+\frac{\rho I_{\text {con }}}{l}\left(\frac{\mathrm{d} \mathbf{N}_{w}(\xi)}{\mathrm{d} \xi}\right)^{T} \\
\cdot\left(\frac{\mathrm{d} \mathbf{N}_{w}(\xi)}{\mathrm{d} \xi}\right)+\rho A_{\text {con }} l \mathbf{N}_{u}^{T}(\xi) \mathbf{N}_{u}(\xi) \mathrm{d} \xi \\
\Delta \mathbf{K}_{\text {tende }}=\int_{0}^{1}-\frac{E_{\text {tend }} A_{\text {tend }} y_{\text {tend }} \sqrt{1+y_{\text {tend }, x}^{2}}}{l^{2}}\left(\frac{\mathrm{d} \mathbf{N}_{u}(\xi)}{\mathrm{d} \xi}\right)^{T} \\
\cdot\left(\frac{\mathrm{d}^{2} \mathbf{N}_{w}(\xi)}{\mathrm{d} \xi^{2}}\right) \mathrm{d} \xi,
\end{gathered}
$$$$
\text { where } A_{\text {tend }} \text { is the area of tendons. }
$$

\section{B. Explicit Expressions for the Characteristic Matrices in (12)}

$$
\begin{aligned}
& \mathbf{M}_{v i}=\left[\begin{array}{cccc}
m_{v i} & 0 & 0 & 0 \\
0 & J_{\theta i} & 0 & 0 \\
0 & 0 & m_{b(2 i-1)} & 0 \\
0 & 0 & 0 & m_{b 2 i}
\end{array}\right] \\
& \mathbf{C}_{v i}=\left[\begin{array}{cccc}
C_{s(2 i-1)}+C_{s 2 i} & -C_{s(2 i-1)} e_{(2 i-1)}+C_{s 2 i} e_{2 i} & -C_{s(2 i-1)} & -C_{s 2 i} \\
-C_{s(2 i-1)} e_{(2 i-1)}+C_{s 2 i} e_{2 i} & C_{s(2 i-1)} e_{(2 i-1)}^{2}+C_{s 2 i} e_{2 i}^{2} & C_{s(2 i-1)} e_{(2 i-1)} & -C_{s 2 i} e_{2 i} \\
-C_{s(2 i-1)} & C_{s(2 i-1)} e_{(2 i-1)} & C_{s(2 i-1)} & 0 \\
-C_{s 2 i} & -C_{s 2 i} e_{2 i} & 0 & C_{s 2 i}
\end{array}\right], \\
& \mathbf{K}_{v i}=\left[\begin{array}{cccc}
K_{s(2 i-1)}+K_{s 2 i} & -K_{s(2 i-1)} e_{(2 i-1)}+K_{s 2 i} e_{2 i} & -K_{s(2 i-1)} & -K_{s 2 i} \\
-K_{s(2 i-1)} e_{(2 i-1)}+K_{s 2 i} e_{2 i} & K_{s(2 i-1)} e_{(2 i-1)}^{2}+K_{s 2 i} e_{2 i}^{2} & K_{s(2 i-1)} e_{(2 i-1)} & -K_{s 2 i} e_{2 i} \\
-K_{s(2 i-1)} & K_{s(2 i-1)} e_{(2 i-1)} & K_{s(2 i-1)} & 0 \\
-K_{s 2 i} & -K_{s 2 i} e_{2 i} & 0 & K_{s 2 i}
\end{array}\right],
\end{aligned}
$$


where $K_{s(2 i-1)}, K_{s 2 i}, C_{s(2 i-1)}$, and $C_{s 2 i}$ are the stiffness and the damping of the two bogies.

\section{Explicit Expressions for the Characteristic Matrices in (14a)-(14c)}

$$
\begin{aligned}
& \mathbf{M}_{s}=\left[\begin{array}{cccc}
\mathbf{M}_{\text {con }} & \mathbf{0} & \cdots & \mathbf{0} \\
\mathbf{0} & \mathbf{M}_{v 1} & \cdots & \mathbf{0} \\
\vdots & \vdots & \ddots & \vdots \\
\mathbf{0} & \mathbf{0} & \cdots & \mathbf{M}_{v N_{v}}
\end{array}\right], \\
& \mathbf{C}_{s}=\left[\begin{array}{cc}
\mathbf{C}_{\mathrm{con}}+\mathbf{C}_{t} & -\mathbf{C}_{\mathrm{con} v} \\
-\mathbf{C}_{\mathrm{con} v}^{T} & \mathbf{C}_{v}
\end{array}\right], \\
& \mathbf{C}_{t}=\sum_{i=1}^{2 N_{v}} C_{t i} \mathbf{A}_{e_{i}}^{T} \mathbf{N}_{w}^{T}\left(\xi_{i}\right) \mathbf{N}_{w}\left(\xi_{i}\right) \mathbf{A}_{e_{i}}, \\
& \mathbf{C}_{\text {con } v}=\sum_{i=1}^{2 N_{v}} C_{t i} \mathbf{A}_{e_{i}}^{T} \mathbf{N}_{w}^{T}\left(\xi_{i}\right) \mathbf{B}_{i}, \\
& \mathbf{K}_{s}=\left[\begin{array}{cc}
\mathbf{K}_{\mathrm{con}}+\Delta \mathbf{K}_{\mathrm{tend}}+\mathbf{K}_{t}+v \mathbf{K}_{c d} & -\mathbf{K}_{\mathrm{con} v} \\
-\left(\mathbf{K}_{\mathrm{con} v}+v \mathbf{K}_{c v}\right)^{T} & \mathbf{K}_{v}
\end{array}\right], \\
& \mathbf{K}_{t}=\sum_{i=1}^{2 N_{v}} K_{t i} \mathbf{A}_{e_{i}}^{T} \mathbf{N}_{w}^{T}\left(\xi_{i}\right) \mathbf{N}_{w}\left(\xi_{i}\right) \mathbf{A}_{e_{i}}, \\
& \mathbf{K}_{c d}=\sum_{i=1}^{2 N_{v}} C_{t i} \mathbf{A}_{e_{i}}^{T} \mathbf{N}_{w}^{T}\left(\xi_{i}\right) \frac{\mathrm{d} \mathbf{N}_{w}\left(\xi_{i}\right)}{\mathrm{d} \xi} \mathbf{A}_{e_{i}}, \\
& \mathbf{K}_{B v}=\sum_{i=1}^{2 N_{v}} K_{t i} \mathbf{A}_{e_{i}}^{T} \mathbf{N}_{w}^{T}\left(\xi_{i}\right) \mathbf{B}_{i}, \\
& \mathbf{K}_{c v}=\sum_{i=1}^{2 N_{v}} C_{t i} \mathbf{A}_{e_{i}}^{T} \frac{\mathrm{d} \mathbf{N}_{w}^{T}\left(\xi_{i}\right)}{\mathrm{d} \xi} \mathbf{B}_{i}, \\
& \mathbf{C}_{v}=\left[\begin{array}{cccc}
\mathbf{C}_{v 1} & \mathbf{0} & \cdots & \mathbf{0} \\
\mathbf{0} & \mathbf{C}_{v 2} & \cdots & \mathbf{0} \\
\vdots & \vdots & \ddots & \vdots \\
\mathbf{0} & \mathbf{0} & \cdots & \mathbf{C}_{v N_{v}}
\end{array}\right], \\
& \mathbf{K}_{v}=\left[\begin{array}{cccc}
\mathbf{K}_{v 1} & \mathbf{0} & \cdots & \mathbf{0} \\
\mathbf{0} & \mathbf{K}_{v 2} & \cdots & \mathbf{0} \\
\vdots & \vdots & \ddots & \vdots \\
\mathbf{0} & \mathbf{0} & \cdots & \mathbf{K}_{v N_{v}}
\end{array}\right],
\end{aligned}
$$




$$
\begin{gathered}
j \text { th column } \\
\mathbf{B}_{i}=\left[\begin{array}{llll}
0 \cdots 0 & 1 & 0 \cdots 0
\end{array}\right]_{1 \times 4 N_{v}}
\end{gathered} \quad, \quad j= \begin{cases}2 i+1 & \text { if } i \text { is an odd number; } \\
2 i & \text { if } i \text { is an even number. }\end{cases}
$$

\section{Competing Interests}

The author declares that they have no competing interests.

\section{Acknowledgments}

This research is sponsored by the National Natural Science Foundation of China (51508155) and the Natural Science Foundation of Jiangsu Province, China (BK20130844).

\section{References}

[1] A. Ariaei, S. Ziaei-Rad, and M. Ghayour, "Vibration analysis of beams with open and breathing cracks subjected to moving masses," Journal of Sound and Vibration, vol. 326, no. 3-5, pp. 709-724, 2009.

[2] U. Andreaus, P. Casini, and F. Vestroni, "Non-linear dynamics of a cracked cantilever beam under harmonic excitation," International Journal of Non-Linear Mechanics, vol. 42, no. 3, pp. 566-575, 2007.

[3] M.-H. H. Shen and Y. C. Chu, "Vibrations of beams with a fatigue crack," Computers and Structures, vol. 45, no. 1, pp. 7993, 1992.

[4] A. D. Dimarogonas, S. A. Paipetis, and T. G. Chondros, Analytical Methods in Rotor Dynamics, vol. 9 of Mechanisms and Machine Science, Springer, Dordrecht, The Netherlands, 2nd edition, 2013

[5] S. Caddemi, I. Caliò, and M. Marletta, "The non-linear dynamic response of the Euler-Bernoulli beam with an arbitrary number of switching cracks," International Journal of Non-Linear Mechanics, vol. 45, no. 7, pp. 714-726, 2010.

[6] D. P. Patil and S. K. Maiti, "Detection of multiple cracks using frequency measurements," Engineering Fracture Mechanics, vol. 70, no. 12, pp. 1553-1572, 2003.

[7] B. Biondi and S. Caddemi, "Closed form solutions of EulerBernoulli beams with singularities," International Journal of Solids and Structures, vol. 42, no. 9-10, pp. 3027-3044, 2005.

[8] S. Caddemi and I. Caliò, "Exact solution of the multi-cracked Euler-Bernoulli column," International Journal of Solids and Structures, vol. 45, no. 5, pp. 1332-1351, 2008.

[9] S. S. Law and X. Q. Zhu, "Dynamic behavior of damaged concrete bridge structures under moving vehicular loads," Engineering Structures, vol. 26, no. 9, pp. 1279-1293, 2004.

[10] V. Jaksic, A. O'Connor, and V. Pakrashi, "Damage detection and calibration from beam-moving oscillator interaction employing surface roughness," Journal of Sound and Vibration, no. 17, pp. 3917-3930, 2014.

[11] C. Fu, "The effect of switching cracks on the vibration of a continuous beam bridge subjected to moving vehicles," Journal of Sound and Vibration, vol. 339, pp. 157-175, 2015.

[12] F. S. Buezas, M. B. Rosales, and C. P. Filipich, "Damage detection with genetic algorithms taking into account a crack contact model," Engineering Fracture Mechanics, vol. 78, no. 4, pp. 695712, 2011

[13] R. Ruotolo, C. Surace, P. Crespo, and D. Storer, "Harmonic analysis of the vibrations of a cantilevered beam with a closing crack," Computers and Structures, vol. 61, no. 6, pp. 1057-1074, 1996.

[14] K. V. Nguyen, "Comparison studies of open and breathing crack detections of a beam-like bridge subjected to a moving vehicle," Engineering Structures, vol. 51, pp. 306-314, 2013.

[15] M. Saiidi, B. Douglas, and S. Feng, "Prestress force effect on vibration frequency of concrete bridges," Journal of Structural Engineering, vol. 120, no. 7, pp. 2233-2241, 1994.

[16] K. Kanaka and G. Venkateswara, "Free vibration behavior of prestressed beams," Journal of Structural Engineering (ASCE), vol. 112, no. 2, pp. 433-437, 1986.

[17] A. Miyamoto, K. Tei, H. Nakamura, and J. W. Bull, "Behavior of prestressed beam strengthened with external tendons," Journal of Structural Engineering (ASCE), vol. 126, no. 9, pp. 1033-1044, 2000.

[18] E. Hamed and Y. Frostig, "Natural frequencies of bonded and unbonded prestressed beams-prestress force effects," Journal of Sound and Vibration, vol. 295, no. 1-2, pp. 28-39, 2006.

[19] O. R. Jaiswal, "Effect of prestressing on the first flexural natural frequency of beams," Structural Engineering and Mechanics, vol. 28, no. 5, pp. 515-524, 2008.

[20] D. L. Logan, A First Course in the Finite Element Method, Thomson, Toronto, Canada, 4th edition, 2007.

[21] R. W. Clough and J. Penzien, Dynamics of Structures, McGrawHill, New York, NY, USA, 2nd edition, 1993.

[22] M. M. Abdel Wahab, G. De Roeck, and B. Peeters, "Parameterization of damage in reinforced concrete structures using model updating," Journal of Sound and Vibration, vol. 228, no. 4, pp. 717-730, 1999. 


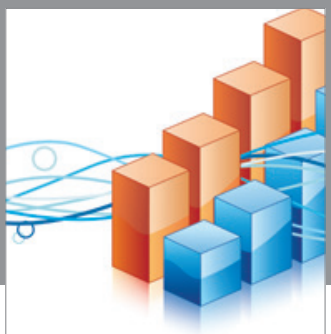

Advances in

Operations Research

vatem alat4

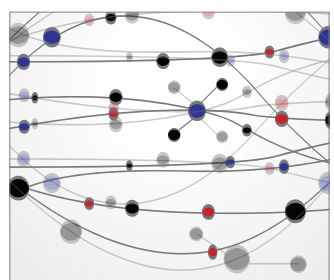

\section{The Scientific} World Journal
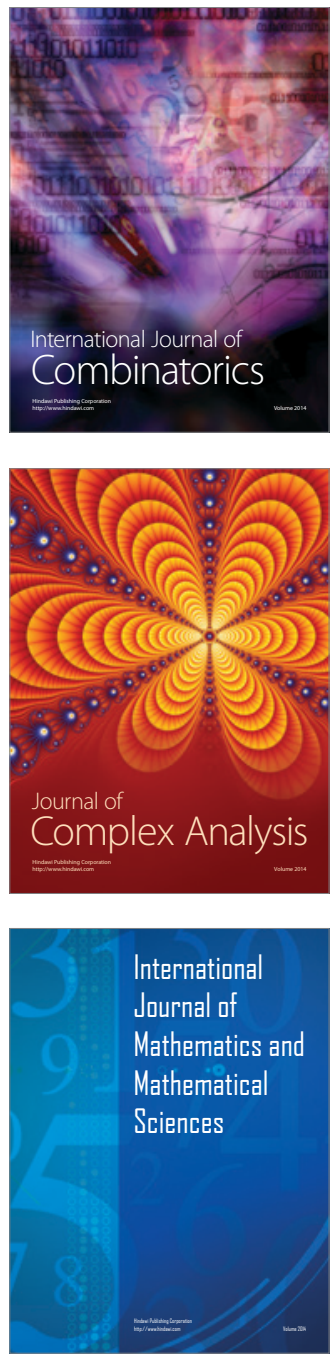
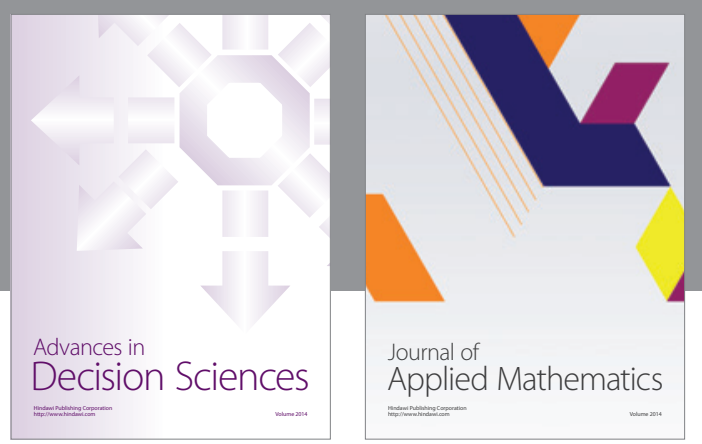

Algebra

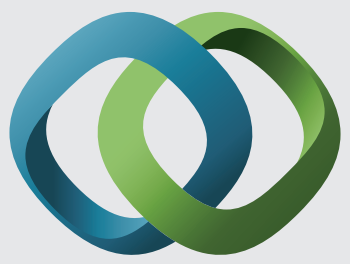

\section{Hindawi}

Submit your manuscripts at

http://www.hindawi.com
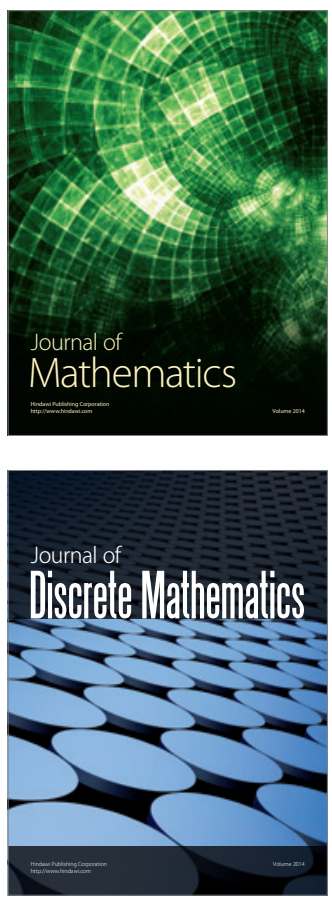

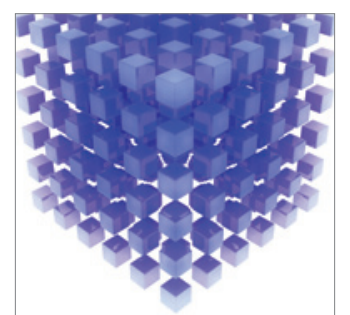

Mathematical Problems in Engineering
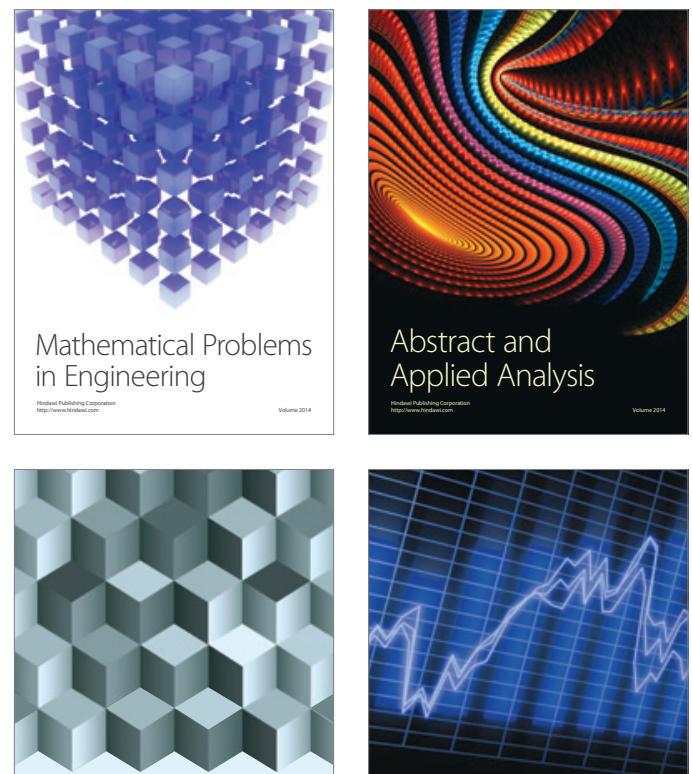

Journal of

Function Spaces

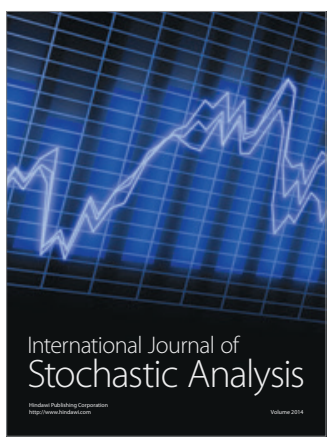

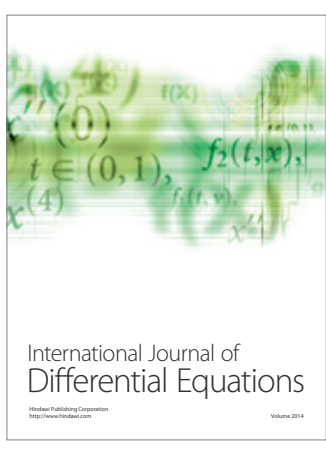
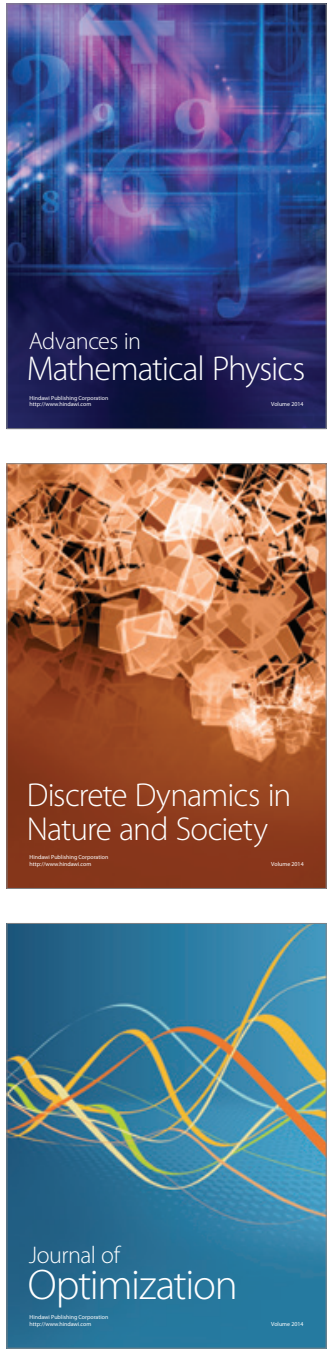